\title{
A Estratégia de Saúde da Família no controle da tuberculose em Curitiba (PR)
}

\author{
Family Health Strategy in tuberculosis control \\ in Curitiba, State of Paraná
}

Janete Marquieviz ${ }^{1}$ Ilcelia dos Santos Alves ${ }^{1}$ Eduardo Borba Neves ${ }^{1}$ Leandra Ulbricht ${ }^{2}$
${ }^{1}$ Centro Universitário Campos de Andrade. R. João Scuissiato ${ }^{\circ} 1$, Santa Quitéria. 80.310-310 borbaneves@hotmail.com ${ }^{2}$ Departamento Acadêmico de Educação Física, Universidade Tecnológica Federal do Paraná.

\begin{abstract}
Tuberculosis defined by some as the 'neglected calamity' is still an important public health problem. To try to improve results in tackling the disease, actions have been decentralized to the level of Primary Health Care (PHC), which has been demanding a new direction in the Family Health Strategy (FHS). This research sought to analyze the evolution of the FHS in the municipality of Curitiba between the years 2000 and 2009 and its impact on incidence rates of tuberculosis. The aggregate-type ecological study with a longitudinal territorial time-series base was the methodology used. Data collection was conducted from October 2010 to July 2011. The main results revealed a marked increase of $127.63 \%$ in the number of Family Health Teams, with a $76.28 \%$ increase of coverage. There was also concern about the ongoing training of these teams, which reflected positively in increasing the number of diagnostic tests performed, reducing the number of new cases, the proportion of individuals abandoning treatment and the mortality rate related to tuberculosis. From the research conducted it can be seen that there has been a correction in the direction of actions to control the incidence of tuberculosis in the municipality of Curitiba.
\end{abstract}

Key words Tuberculosis, Family Health Program, Primary Health Care
Resumo A tuberculose, definida por alguns como a 'calamidade negligenciada, é ainda um importante problema de saúde pública. Para tentar melhorar os resultados no seu enfrentamento, as ações foram descentralizadas para a Atenção Primária à Saúde (APS), o que vem demandando uma nova orientação na Estratégia de Saúde da Família (ESF). Esta pesquisa teve como objetivo analisar a evolução da Estratégia de Saúde da Família (ESF) no município de Curitiba entre os anos de 2000 a 2009 e seus reflexos sobre os casos de Tuberculose. Como metodologia utilizou-se o estudo Ecológico tipo agregado de base territorial longitudinal de séries temporais. A coleta de dados foi realizada no período de outubro de 2010 a julho de 2011. Como principais resultados verificou-se um aumento expressivo de 127,63\% no número de Equipes de Saúde da Família, com um aumento da cobertura em 76,28\%. Existiu também uma preocupação com relação à capacitação continuada destas equipes o que repercutiu de forma positiva no aumento de exames para diagnóstico realizado, redução do número de casos novos, redução da proporção de abandono do tratamento e da taxa de mortalidade relacionada a tuberculose. Pelo estudo realizado percebe-se uma correção no direcionamento das ações de controle da Tuberculose no município de Curitiba.

Palavras-chave Tuberculose, Programa Saúde da Família, Atenção Primária à Saúde 


\section{Introdução}

A tuberculose é um problema de saúde pública que se mantém nos países em desenvolvimento como uma das mais importantes causas de mortalidade entre adultos jovens (entre 15 e 49 anos) ${ }^{1-2}$.

Apesar dos avanços conseguidos no combate a esta doença, o Brasil é um dos 22 países priorizados pela Organização Mundial de Saúde (OMS), pois ele faz parte do grupo que abrange $80 \%$ da carga mundial de Tuberculose. Atualmente o país está na $19^{a}$ posição (já tendo ocupado a $14^{\text {a }}$ em 2004) com um total de 71.700 casos novos da doença notificados em 2009, apresentando uma taxa de incidência de 37 por cada grupo de 100 mil habitantes. Desde 1990, a taxa de incidência apresenta uma queda de $26 \%$, representando aproximadamente $1,4 \%$ ao ano ${ }^{3}$.

A taxa de mortalidade por tuberculose no Brasil teve uma redução de $16,7 \%$ entre os anos de 2002 a 2008, passando de três casos para 2,5 por 100 mil habitantes. Contudo, destaca-se que anualmente ainda ocorrem cerca de 4.700 óbitos devido à tuberculose, doença curável e evitável ${ }^{3}$.

Para se conseguir avanços na intercepção desta doença, a estratégia utilizada em seu combate não pode mais ser centralizada em ações curativas, fazse necessário uma abordagem mais ampla que vai desde o combate à miséria, a qual dá suporte à doença ${ }^{4}$ até a reorientação da Atenção Primária à Saúde (APS), incorporando ações e medidas em programas como o de Saúde da Família (PSF) e Agentes Comunitários de Saúde (PACS), onde se espera a melhoria do diagnóstico precoce e a realização da supervisão do tratamento (impactando na redução das taxas de abandono $)^{5}$.

No Brasil a distribuição geográfica da tuberculose concentra-se em grandes centros urbanos (ou seja, em municípios que abrigam capitais e regiões metropolitanas $)^{6}$, assim torna-se relevante estudar municípios que atendam estas condições como o de Curitiba (que faz parte da Região Metropolitana de Curitiba e que também é a capital do Estado do Paraná) e que tem reconhecido a tuberculose (TB) como um agravo prioritário para a gestão municipal devido a sua importância no contexto de saúde pública e ser, entre os agravos de notificação, uma das doenças infecto-contagiosas com maior morbimortalidade no município ${ }^{7}$.

Neste sentido, o presente estudo teve por objetivo analisar a evolução do Programa Saúde da Família no município de Curitiba entre os anos de 2000 a 2009 e seus reflexos sobre os casos de Tuberculose na mesma cidade.

\section{Métodos}

O campo de estudo consistiu no município de Curitiba-PR, contando com uma população de aproximadamente 1.900 .000 habitantes. Utilizouse o estudo Ecológico tipo agregado de base territorial (município de Curitiba) longitudinal de séries temporais, onde a mesma área foi investigada durante os últimos 10 anos (de 2000 a 2009) ${ }^{8}$.

Foram utilizados dados disponibilizados pela Secretaria Municipal de Saúde de Curitiba e informações colhidas em pesquisa documental nos Planos Operativos Anuais, relatórios de programas e portarias da SMS de Curitiba e relatórios de atividades realizadas, referentes ao período descrito. A coleta de dados foi realizada no período de outubro de 2010 a julho de 2011.

\section{Resultados}

No período estudado observa-se pela Tabela 1, um aumento expressivo de 127,63\% no número de Equipes de Saúde da Família e um aumento menor $(43,24 \%)$ no número de Unidades de Saúde que contavam com estas equipes, bem como no número de ACS (aumento de 27,88\%). Sendo que o ano de 2000 foi marcado pela transformação de todas as Unidades de Saúde do Distrito Sanitário Bairro Novo, em PSF.

Houve também um acréscimo no número de pessoas atendidas pelo PSF em 76,28\%, o que já era esperado devido ao aumento de $14,39 \%$ da população no município (1.618.279 habitantes em 2000 para 1.851.215 em 2009).

Pode-se verificar que a notificação de casos novos de tuberculose caiu 19,21\%, contudo acompanhando-se os relatórios de Baciloscopias (implantado a partir de 2005), nota-se que o número de culturas para diagnóstico tem se elevado ao longo do período (de 8646 em 2005 para 13569 em 2009) demonstrando a preocupação do município em identificar casos novos da doença.

A proporção de abandono do tratamento também está em redução (13,72 em 2001 para 11,50 em 2009), o que acaba repercutindo de forma positiva em relação a redução visualizada no número de óbitos relacionados à tuberculose (queda de $48,57 \%$ ), bem como na diminuição da taxa de mortalidade por tuberculose (queda de $55,04 \%)$. 


\section{Discussão}

Para o controle da tuberculose as diretrizes repassadas pelo Plano Nacional de Controle a Tuberculose indicavam que era necessário detectar $90 \%$ dos casos esperados e curar pelo menos $85 \%$ dos diagnosticados. É necessário ainda, manter o abandono abaixo de $5 \%$ e expandir o tratamento supervisionado (observação direta da tomada de medicação para TB) para $100 \%$ das Unidades de Saúde dos municípios prioritários. Manter registro atualizado de casos diagnosticados e encerramento em 100\% destes. Realizar a busca dos sintomáticos respiratórios e encaminhar 100\% dos contatos domiciliares de um caso novo, ofertando-se ainda o teste anti-HIV para $100 \%$ dos adultos com $\mathrm{TB}^{5,6,9,10}$.

Em Curitiba pode-se verificar um aumento expressivo de 127,63\% no número de Equipes de Saúde da Família e um aumento menor no número de Unidades de Saúde que contavam com estas equipes, bem como no número de ACS.

A partir do momento em que as ações de controle da TB passaram a fazer parte das ações de Atenção Básica, ou seja, de responsabilidade municipal, os municípios passaram então a planejar e a executar as atividades de controle da TB com autonomia, evidenciando-se o processo de descentralização política e administrativa do país. Alguns autores acreditam que essa política foi de suma importância, pois deu autonomia aos municípios para desenvolverem as ações de promoção e prevenção do agravo, sendo, portanto favorável à implantação e sustentabilidade da estratégia DOTS (Tratamento Diretamente Observado de Curta Duração $)^{4,11}$.

Em Curitiba pode-se visualizar que houve um acréscimo na cobertura do atendimento com um aumento $76,28 \%$ no número de pessoas atendidas pelo PSF e uma redução no número de óbitos relacionados à tuberculose (queda de 48,57\%) e na taxa de mortalidade por tuberculose (queda de 55,04\%), assim como nos abandonos ao tratamento (queda de $36,27 \%$ ), no período estudado.

Outros autores ${ }^{12}$ discordam, pois descrevem que esta estratégia de descentralização tem apresentado nos países em desenvolvimento um limitado impacto na redução das taxas de incidência, principalmente em grandes metrópoles que tenham uma elevada prevalência de infecção por HIV e alta desigualdade em saúde. E descrevem ainda, que no Brasil a descentralização das ações para o PSF não apresentou um bom desempenho no acesso ao diagnóstico ${ }^{12}$.

Assim, em Curitiba os dados parecem concordar com os primeiros autores, ao demonstrarem uma adequação do direcionamento da estratégia de combate à tuberculose, pois se verifica um aumento de cobertura e redução tanto na proporção de abandono do tratamento como no número de óbitos relacionados com a TB e na taxa de mortalidade por TB.

Importante ressaltar a redução da notificação de casos novos de tuberculose (queda de 19,21\%), mesmo com a elevação do número de exames para diagnóstico.

Uma Pesquisa realizada em Bayeux (PB) aponta alguns desafios importantes para a resolutividade da atenção como a realização da coleta de escarro pelas equipes do PSF, entendida como um dos procedimentos prioritários para a efetivação do diagnóstico; fornecimento do valetransporte aos pacientes (para promover maior adesão ao tratamento); adoção da visita domiciliar por todos os profissionais das equipes do PSF como rotina de trabalho e atendimento do

Tabela 1. Série histórica da Tuberculose no Município de Curitiba de 2000 a 2009

\begin{tabular}{lcccccccccc}
\hline \multicolumn{1}{c}{ Ano } & $\mathbf{2 0 0 0}$ & $\mathbf{2 0 0 1}$ & $\mathbf{2 0 0 2}$ & $\mathbf{2 0 0 3}$ & $\mathbf{2 0 0 4}$ & $\mathbf{2 0 0 5}$ & $\mathbf{2 0 0 6}$ & $\mathbf{2 0 0 7}$ & $\mathbf{2 0 0 8}$ & $\mathbf{2 0 0 9}$ \\
\hline No de US/PSF & 37 & 42 & 42 & 42 & 43 & 45 & 49 & 52 & 53 & 53 \\
No de equipes PSF & 76 & 117 & 116 & 131 & 113 & 129 & 157 & 165 & 166 & 173 \\
No de ACS & 800 & 830 & 830 & 997 & 1140 & 1139 & 1202 & 1110 & 1097 & 1023 \\
Pop. atendida no PSF & 342.000 & 526.500 & 400.200 & 451.950 & 389.850 & 445.050 & 541.650 & 572.700 & 612.986 & 602.865 \\
No casos novos TB & 557 & 488 & 553 & 531 & 483 & 539 & 458 & 457 & 445 & 450 \\
N $^{\circ}$ óbitos relac. a TB & 35 & 25 & 36 & 34 & 27 & 27 & 34 & 25 & 17 & 18 \\
No de abandonos ao tratamento $^{\circ}$ & $\mathbf{\ldots}$ & 102 & 108 & 93 & 78 & 70 & 69 & 69 & 83 & 65 \\
Tx Mortal Tb (1000) & 216,28 & 154,3 & 218,9 & 203,45 & 156,34 & 153,59 & 190,1 & 139,09 & 92,99 & 97,23 \\
\hline
\end{tabular}

... Informação não registrada pela Secretaria Municipal de Saúde de Curitiba.

Fonte: Ministério da Saúde e Secretaria Municipal de Saúde de Curitiba 
paciente de TB no horário de almoço (o que evidenciou um problema de acesso às unidades pelos relatos dos pacientes que trabalhavam $)^{13}$.

Com relação ao financiamento, em 2000, no Brasil, os gastos foram de US\$ 7,97 per capita em atenção primária à saúde, enquanto para a TB foram de US\$ 0,13. A partir de outubro de 2001, "o incentivo para alta curada do paciente com Tuberculose" estava no Bloco de "Ações Assistenciais Estratégicas”, voltadas para grupos populacionais considerados prioritários pelo MS A partir de 2002, ocorrem reformulações nos valores de incentivos relacionados à alta por cura da TB, fixando novos valores do incentivo para o controle da TB para a notificação de casos novos, para alta por cura quando o tratamento for autoadministrado e alta por cura quando o tratamento for supervisionado. Em 2003, o Ministério da Saúde elegeu a TB como um problema de saúde pública a ser combatido e ampliando o orçamento do Programa Nacional de Controle da Tuberculose (PNCT) 3 . Em 2004, o critério de concessão de bônus foi substituído por uma nova sistemática implantada pelo MS a partir de um recurso anual concedido mediante a apresentação de um plano de trabalho elaborado pelos municípios ${ }^{14}$.

O município de Curitiba ${ }^{15}$ recebeu verba do Ministério da Saúde específica para Intensificação das ações de vigilância de controle da Tuberculose nos anos de 2003 pela Portaria 2305 de 04/ $12 / 03$. Nesse ano verificou-se o maior percentual de notificação de casos novos no período (635 novos casos), percentual este que foi baixando até o final do período estudado.

De 2004 até 2006, o município de Curitiba recebeu verba do MS, específica para intensificação das ações de vigilância de controle da TB, pelas Portarias 2405 de 05/11/04; 543 de 08/04/05 e 3162 de $14 / 12 / 06^{15}$.

Esses recursos foram utilizados para treinamentos, palestras e capacitações, aos trabalhadores da saúde que realizam ações no controle do agravo, confecção de folders e cartazes, distribuídos nos serviços de saúde do município ${ }^{16}$. A partir daí não foram encontrados outros repasses para o fundo Municipal de saúde, destinados especificamente às ações de vigilância no controle da TB.

Assim, os repasses realizados pelo Ministério da Saúde, foram aplicados em Curitiba nas ações de promoção e prevenção e controle da doença destacando assim a importância do incentivo financeiro para que o município possa realizar ações no controle do agravo.
Para fortalecer as ações com relação ao diagnóstico precoce, em 2006 iniciou-se um trabalho de parceria com o Programa Saúde Mental do município no trato do paciente com comorbidades como dependência de álcool e outras drogas associadas à tuberculose. Com expansão da parceria ao CAPS (Centro de Atenção Psicossocial), no sentido da identificação dos sintomáticos respiratórios e auxílio na adesão do tratamento da $\mathrm{TB}^{15}$.

Pesquisa realizada em Belo Horizonte demonstrou que segundo a percepção dos pacientes, os principais motivos do abandono do tratamento foram o uso das drogas e bebidas alcoólicas $(30 \%)$ e a melhora clínica $(25 \%)^{10}$. Tendo o uso de álcool e outras drogas, como facilitadores para contrair a TB, é importante a parceria entre os setores que atendem essa demanda, que muitas vezes só comparecem nas Unidades de Saúde para buscar medicações pertinentes a Saúde Mental. Sendo assim um ótimo momento para a busca de sintomáticos respiratórios.

Em 2007, foi realizada uma oficina de supervisão do programa informatizado da Epidemiologia dos Distritos, Unidades de Saúde, Centro de Atenção à Saúde, Centro de Epidemiologia e Suporte Técnico de Informática. Realizado treinamento em tuberculose para médicos e enfermeiros dos Centros Municipais de Urgências Médicas (CMUM), numa parceria com a secretaria do estado da saúde, o treinamento foi focado na epidemiologia da TB ${ }^{15}$. É fundamental a designação de profissionais capacitados e que possuam habilidades, tanto gerencial quanto técnica, para assumir a coordenação dos PCT nos municípios ${ }^{11}$.

A informática tem um papel importante em Curitiba para controle dos casos de TB no município, podendo assim, os profissionais de todas as Unidades de Saúde, ter acesso ao sistema para registro, notificação e acompanhamento dos casos de TB. Esta preocupação pode ter impactado de forma positiva a redução da proporção do abandono do tratamento no município pesquisado.

Em relação aos treinamentos, são de suma importância para que os profissionais que atuam na atenção primária estejam capacitados para diagnosticarem e tratarem de maneira correta o mais precocemente os portadores de TB, diminuindo a taxa de incidência da doença.

Esta preocupação com a formação continuada dos profissionais de saúde tem sido constante desde 2007, quando 305 profissionais entre médicos e enfermeiros dos Centros Municipais de Urgências Médicas, foram capacitados em tu- 
berculose em parceria com a Secretaria Estadual de Saúde (SESA) ${ }^{17}$.

Em 2008, o treinamento avançou para médicos gineco-obstetras e enfermeiros das unidades de saúde de três diferentes distritos sanitários, abordando temas como epidemiologia, diagnóstico e tratamento da TB em gestantes, totalizando 70 profissionais em uma primeira etapa e 120 profissionais na segunda ${ }^{18}$. Ainda no mesmo ano, ocorreu a capacitação multiprofissional em TB e multirresistência para 562 profissionais de saúde do município de Curitiba e região metropolitana, também com uma parceria com a SESA, incluindo o conteúdo de Epidemiologia global, diagnóstico e tratamento ${ }^{18}$. Além disso, durante as atividades relativas à Semana Mundial de Combate a Tuberculose de 2008, o tema foi abordado em sala de espera das Unidades de Saúde e foram realizadas varreduras de sintomáticos respiratórios (SR) e coletas para exames de escarro em áreas de risco, feiras de saúde, asilos, delegacias, casas de prostituição, entre outras ${ }^{15}$.

No ano seguinte, os novos farmacêuticos contratados pela SMS foram capacitados, totalizando 50 profissionais; e também repetido o treinamento em TB multirresistente, para as coordenações municipais e serviços de referência do Estado do Paraná totalizando 120 profissionais ${ }^{19}$. Ainda, em 2009, foram capacitados 688 profissionais de saúde, com ênfase na coinfecção HIV/ $\mathrm{TB}$, prova de tuberculina e quimioprofilaxia como redutores de óbitos por TB, contando com a presença do palestrante convidado Antonio Ruffino Neto ${ }^{19}$. E ainda, ao final deste ano, foram capacitados 700 profissionais para a implementação do novo tratamento para TB no Brasil, com a dose fixa combinada de quatro medicamentos em um ${ }^{19,20}$.

A capacitação constante deve fazer parte de qualquer programa de saúde, em especial os de TB onde se espera que os profissionais envolvidos possam realizar a busca ativa identificando na comunidade (por meio de visitas domiciliares) os indivíduos que sejam sintomáticos respiratórios (apresentem tosse por três semanas ou mais); que sejam capazes de orientar as famílias da importância de acompanhar a tomada dos medicamentos pelos pacientes e que realizem reuniões educativas com as comunidades ${ }^{1}$.

Estudo realizado em Vitória (ES) aponta a importância do treinamento constante das equipes. O estudo realizado com médicos e enfermeiros identificou um grande número de respostas inadequadas em relação à transmissão da doença por pessoas que estão apenas infectadas (o que poderia ser esclarecido por qualquer manual de controle da doença); quanto à escolha do local de tratamento enquanto os enfermeiros responderam que deveria vir por parte do paciente, notou-se uma resistência dos médicos em respeitar esta escolha (o que poderia levar a um comprometimento da adesão ao tratamento supervisionado $)^{1}$.

Estudo realizado com ACS mostrou que os mesmos tinham um conhecimento limitado para reconhecer a sintomatologia clássica do sintomático respiratório (tosse prolongada) onde apenas 3 de 105 souberam apresentar uma definição correta do termo. Além disso, o sintoma que recebe menor destaque nas cartilhas a febre, foi o mais referido por estes profissionais (em lugar da tosse prolongada e falta de apetite/emagrecimento); e igualmente preocupante foi o desconhecimento com relação ao processo de disseminação da doença ${ }^{5}$.

É importante destacar que os programas investem em capacitações e que mostram resultados positivos, apresentam um impacto positivo quanto aos custos gerados por esta doença tanto para as famílias, quanto para os pacientes. Estudo realizado em Salvador demonstrou que as famílias arcaram com 33\% dos custos totais da $\mathrm{TB}$, sendo que o custo médio variava de $\mathrm{R} \$ 481,03$ (do paciente diagnosticado pela primeira vez e tratado ambulatoriamente por seis meses) até $\mathrm{R} \$ 603,23$ (para a família do paciente com TB multiresistente). Já o setor público arcava com $62 \%$ (sem computar as despesas com a vigilância epidemiológica e campanhas educacionais). Sendo que o custo médio de um caso novo era de $\mathrm{R} \$ 185,93$ e para o retratamento de $\mathrm{R} \$ 207,70$. Os autores chamam a atenção para o fato dos gastos públicos terem sido direcionados para internamentos $(65 \%)$, enquanto que as atividades de prevenção, busca dos faltosos e profilaxia estavam sendo negligenciadas (somente 3\% dos recursos eram direcionados para estas ações) ${ }^{21}$.

Em 2009, foi realizada a Semana Mundial de Combate a Tuberculose, com atividades educativas em todas as unidades de saúde, palestras em 43 escolas, visita a dez asilos e duas casas de recuperação, varredura de sintomáticos respiratórios em áreas de risco, capacitação em TB com ênfase em coinfecção HIV/TB, realizado vídeo conferência Estadual sobre o novo tratamento da TB para o Brasil, reunião técnica sobre o novo programa informatizado da $\mathrm{TB}$, capacitação para o novo tratamento da TB, entrando em vigor para toda a rede municipal de saúde o novo esquema de tratamento ${ }^{16}$. 
Neste estudo observa-se que, a partir do ano de 2004, as ações de controle da TB no município de Curitiba foram intensificadas, trazendo resultados positivos em relação ao agravo, tanto na taxa de incidência quanto na de mortalidade e abandono.

$\mathrm{O}$ aumento do número de equipes de ESF, o qual necessitou de intervenções políticas para que fosse ampliado, acredita-se que teve papel fundamental no alcance desses resultados nos últimos dez anos. Sendo que até os dias de hoje, Curitiba segue na busca de melhores resultados no controle da TB, monitorando por meio da vigilância epidemiológica, a situação e a tendência da doença, examinando sintomáticos respiratórios através de baciloscopia e cultura para BAAR (Bacilo Álcool Ácido Resistente) no escarro, intensificando o exame de contatos de todos os pacientes de tuberculose; tratando e acompanhando todos os casos diagnosticados pelas equipes das Unidades de Saúde, tendo implantado o tratamento supervisionado para todas as unidades de saúde. Mantém ainda um serviço laboratorial de reconhecida qualidade e sensibilidade pelo Ministério da Saúde; procura proteger os sadios por meio de altas coberturas de vacinação BCG nos menores de um ano; da quimioprofilaxia para os contatos de TB, imunodeprimidos e finalmente com medidas de biossegurança visando à proteção de profissionais da rede e usuários das Unidades de Saúde. A implantação do Vale de Apoio Nutricional vem se mostrando importante ação de apoio às questões sociais associadas. Mas para que o município continue realizando suas ações, precisa continuar recebendo repasses destinados à TB e a ampliação da Estratégia de Saúde da Família.

O PSF está em pleno processo de aprofundamento de suas bases conceituais e criação de uma nova práxis entre os trabalhadores integrantes das equipes. Tal construção ocorre concomitantemente ao seu crescimento quantitativo e ao caráter substitutivo em relação à rede básica atualmente instalada no país. É preciso lembrar, ainda que esse cres- cimento está sendo estimulado por uma grande aceitação popular, haja vista sua aprovação em centenas de Conferências Municipais de Saúde realizadas em todo o país nos últimos anos ${ }^{22}$.

\section{Conclusão}

Pelo estudo realizado percebe-se uma correção no direcionamento das ações de controle da Tuberculose no município de Curitiba.

Foi realizado um investimento no Programa Saúde da Família tanto na capacitação continuada destes profissionais como no aumento do número de equipes, que repercutiu de forma positiva no número de pessoas atendidas, no aumento no número de exames para diagnóstico realizados, redução do número de casos novos, redução da proporção de abandono do tratamento e da taxa de mortalidade relacionada à tuberculose.

Assim, parece que o PSF teve um relevante papel no controle da tuberculose no município, sendo adotado como política pública de atenção primária, atuando não apenas na Unidade de Saúde, mas também no domicílio através do vínculo, da corresponsabilização e do acolhimento realizado pela equipe, facilitando o processo de promoção e prevenção. Além de atuar também, na captação e adesão das pessoas infectadas, fazendo com que pessoas portadoras de tuberculose sejam atendidas e acompanhadas o mais precocemente, evitando assim, transmissão do bacilo a outras pessoas e o abandono ao tratamento.

Espera-se que esse estudo possa contribuir nas ações de gestão em saúde, na reorientação das ações e na incorporação de novas práticas sanitárias através da mensuração do impacto das ações implementadas no controle da tuberculose. E que também contribua com um importante instrumento para gestão e alocação de recursos no que se refere às medidas adotadas para o controle da tuberculose e consequentemente possa melhorar a saúde da população curitibana.

\section{Colaboradores}

J Marquieviz, IS Alves, EB Neves e L Ulbricht participaram igualmente em todas as etapas da pesquisa. 


\section{Referências}

1. Maciel ELN, Araújo WK, Giacomin SS, Jesus FA, Rodrigues PM, Dietze R. O conhecimento de enfermeiros e médicos que trabalham na Estratégia de Saúde da Família acerca da tuberculose no município de Vitória (ES): um estudo de corte transversal. Cien Saude Colet 2009; 14(1):1395-1402.

2. Mascarenhas MDM, Araujo LM, Gomes KRO. Perfil epidemiológico da tuberculose entre casos notificados no Município de Piripiri, Estado do Piauí, Brasil. Epidemiol. Serv. Saúde 2005; 14(1):7-14.

3. Brasil. Ministério da Saúde (MS). Tratamento diretamente observado da tuberculose na atenção básica Protocolo de Enfermagem. Brasília: MS; 2010.

4. Ruffino-Netto AR. Tuberculose: A Calamidade Negligenciada. Revista da Sociedade Brasileira de Medicina Tropical 2002; 35(1):51-58.

5. Maciel ELN, Vieira RCA, Milani EC, Brasil M, Fregona G, Dietze R. O Agente Comunitário de Saúde no Controle da Tuberculose: conhecimentos e Percepções. Cad Saude Publica 2008; 24(6):1377-1386.

6. Hijjar MA, Gerhardt G, Teixeira GM, Procópio MJ. Retrospecto do Controle da Tuberculose no Brasil. Rev Saude Publica 2007; 41(Supl. 1):50-58. Curitiba. Portaria no 2405 de 05/11/04. Curitiba: Secretaria Municipal da Saúde, 2004.

7. Rouquayrl MZ, Almeida Filho N. Epidemiologia e Saúde. 5a. Edição. Rio de Janeiro: Medsi; 1999.

8. Campinas LLSL, Almeida MMMB. Agentes Comunitários de Saúde e o acolhimento aos Doentes com Tuberculose no Programa Saúde da Família. Bol. Pneumol. Sanit. 2004; 12(3):145-154.

9. Paixão LM, Gontijo ED. Perfil de Casos de Tuberculose Notificados e Fatores Associados ao Abandono, Belo Horizonte, MG. Rev Saude Publica 2007; 41(2):205-213.

10. Ruffino-Netto RA, Villa TCS. Implantação dos DOTS em algumas regiões do Brasil, Instituto do Milênio Rede TB, 2006. [acessado 2011 maio 12]. Disponível em: http://www.eerp.usp.br/geotb/Doc/livro_DOTS. pdf

11. Scatena LM. Dificuldades de Acesso a Serviços de Saúde para Diagnóstico de Tuberculose em Municípios do Brasil. Rev Saude Publica 2009; 43(3):389-397.

12. Marcolino ABL. Avaliação do Acesso às Ações de Controle da Tuberculose no Contexto das Equipes de Saúde da Família de Bayeux - PB. Rev Bras Epidemiol 2009; 12(2):144-157

13. Villa TCS, Netto AR, Arcencio RA, Gonzales RIC. As políticas de controle da tuberculose no sistema de saúde no Brasil e a Implantação da Estratégia DOTS (1980 a 2005). In: Ruffino-Netto RA, Villa TCS. (Org.) Implantação dos DOTS em algumas regiões do Brasil, Instituto do Milênio Rede TB, 2006. [acessado 2011 maio 12]. Disponível em: http:// www.eerp.usp.br/geotb/Doc/livro_DOTS.pdf

14. Curitiba. Relatório anual de gestão 2010. Curitiba: Secretaria Municipal da Saúde, 2010. [acessado 2011 05 13]. Disponível em: http://www.saude.curitiba. pr.gov.br/images/institucional/arquivos/relatorios/ relatorio $010 . \mathrm{pdf}$
15. Curitiba. Relatório Final do plano municipal de saúde de Curitiba (2006-2009). Curitiba: Secretaria Municipal da Saúde. 2011. [acessado 2011 jul 11]. Disponível em: http://www.saude.curitiba.pr.gov.br/images/ institucional/arquivos/planos/plano_003.pdf

16. Curitiba. Informe técnico de tuberculose. Curitiba: Secretaria de Vigilância em Saúde; 2010.

17. Curitiba. Relatório de programas da SMS e atividades realizadas no SUS- Curitiba comparativo anual 2006/2007. Acompanhamento do plano operativo anual de 2007 Curitiba: Secretaria Municipal da Saúde. [acessado 2011 maio 13]. Disponível em: http://www.saude.curitiba.pr.gov.br/images/a_ secretaria/arquivos/relatorio_anual_\%202006_ $\% 202007 . p d f$

18. Curitiba. Relatório de programas da SMS e atividades realizadas no SUS-Curitiba comparativo anual 2007/2008. Acompanhamento do plano operativo anual de 2008 Curitiba: Secretaria Municipal da Saúde. [acessado 2011 maio 13]. Disponível em: http://www.saude.curitiba.pr.gov.br/images/a secretaria/arquivos/comparativo_anual_2007_2008 final.pdf

19. Curitiba. Relatório de programas da SMS e atividades realizadas no SUS-Curitiba comparativo anual 2008/2009. Acompanhamento das ações do SUS Curitiba: Secretaria Municipal da Saúde. [acessado 2011 maio 13]. Disponível em: http://www.saude. curitiba.pr.gov.br/images/a_secretaria/arquivos/ relatorio_anual_2008_e_\%202009.pdf

20. Curitiba. Relatório de programas da SMS e atividades realizadas no SUS-Curitiba comparativo anual 2009/2010. Acompanhamento das ações do SUSCuritiba Secretaria Municipal da Saúde. [acessado 2011 maio 13]. Disponível em: http://www.saude. curitiba.pr.gov.br/images/a_secretaria/arquivos/ relatorio_anual_2009 e 2010.pdf

21. Costa JG, Santosa AC, Rodrigues LC, Barreto ML, Roberts JA. Tuberculose em Salvador: Custos para o Sistema de Saúde e para as Famílias. Rev Saude Publica 2005; 39(1):122-128.

22. Duncan BB, Schmidt MI, Giugliani ERJ. Medicina ambulatorial. 3a Edição. Porto Alegre: Artmed; 2006.

Artigo apresentado em 14/03/2012

Aprovado em 20/09/2011

Versão final apresentada em 21/09/2011 